\title{
A Day in the Life of the Laser Communications Relay Demonstration Project
}

\author{
Bernard Edwards, Dave Israel, Armen Caroglanian, and James Spero \\ NASA Goddard Space Flight Center, Greenbelt, MD 20771, USA \\ Tom Roberts \\ Jet Propulsion Laboratory, the California Institute of Technology, Pasadena, CA 91109, USA \\ and \\ John Moores \\ MIT Lincoln Laboratory, Lexington, MA 02420
}

This paper provides an overview of the planned concept of operations for the Laser Communications Relay Demonstration Project (LCRD), a joint project among NASA's Goddard Space Flight Center (GSFC), the Jet Propulsion Laboratory, California Institute of Technology (JPL), and the Massachusetts Institute of Technology Lincoln Laboratory (MIT/LL). LCRD will provide at least two years of bi-directional optical communications at user data rates of up to $1.244 \mathrm{Gbps}$ in an operational environment. The project lays the groundwork for establishing communications architecture and protocols, and developing the communications hardware and support infrastructure, concluding in a demonstration of optical communications' potential to meet NASA's growing need for higher data rates for future science and exploration missions. A pair of flight optical communications terminals will reside on a single commercial communications satellite in geostationary orbit; the two ground optical communications terminals will be located in Southern California and Hawaii. This paper summarizes the current LCRD architecture and key systems for the demonstration, focusing on what it will take to operate an optical communications relay that can support space-to-space, space-to-air, and space-to-ground optical links.

\section{Introduction}

$\mathrm{N}$ ASA has been developing optical communications for both Near Earth and Deep Space applications. Optical communications (or laser communication or "lasercom") is a revolutionary technology that enables NASA to undertake more complex missions in the future that require transmitting more data and/or decreasing the communication system's mass, size, and power burden on the spacecraft:

- For approximately the same mass, power, and volume, an optical communications system will provide significantly higher data rates or data volume than a comparable radio frequency system

- For the same data rate (e.g. 1 Gbps of output), an optical communications system will require less mass, power, and volume than a comparable radio frequency system

Beyond the potential savings in mass, weight, and power, the higher data bandwidth will allow missions with the present day data volumes to operate while requiring less time for communications activities. This savings in communication time will reduce operational constraints for both the spacecraft and the supporting communications network infrastructure.

There exist some differences between the technological approaches to optical communications specifically designed for Near Earth missions versus Deep Space missions. Due to the vastly differing ranges and data rates for Near Earth versus Deep Space missions, some of the technologies applicable to each domain differ in profound ways; however, there are also many technologies which are similar to both! Coordination of system development 
for these two domains maximizes NASA's return on investment. The Laser Communications Relay Demonstration, to launch in 2019, is NASA's flagship optical communications technology demonstration for Near Earth applications. Its purpose is to prove the technology is ready for the Next Generation Tracking and Data Relay Satellites and that it is ready to provide mission critical communications for users.

LCRD will have two optical communications terminals in space on a single Geostationary Earth Orbit (GEO) spacecraft and two optical communications terminals on Earth to allow the mission to demonstrate and test:

- High rate bi-directional communications between Earth and GEO

- Real-time optical relay from Ground Station 1 through the GEO flight payload to Ground Station 2

- Pulse Position Modulations suitable for power limited users, such as small Near Earth missions

- Differential Phase Shift Keying Modulations suitable for Near Earth high data rate communications

- Various mission scenarios through spacecraft simulations at the Earth ground station

- Coding, link layer, and network layer protocols over optical links over an orbiting testbed.

At the same time that it is developing LCRD, NASA is also working on a Next Generation Low Earth Orbit (LEO) User Terminal that is compatible with LCRD. Thus the LCRD flight payload has a requirement to be able to support high rate bi-directional communications between LEO and GEO as well as between Earth and GEO. The current plan is to launch the Next Generation LEO User Terminal to the International Space Station to demonstrate interoperability with LCRD and to demonstrate LEO-to-GEO-to-Earth relay operations.

\section{The Flight Payload}

The LCRD flight payload will be flown on a GEO spacecraft and consists of:

- Two optical communications modules (heads)

- Two optical module controllers

- Two Differential Phase Shift Keying (DPSK) modems that can also support low data rate Pulse Position Modulation (PPM)

- A space switching unit to interconnect the two optical modules and to interface to the host spacecraft

An optical communications terminal on LCRD consists of an optical module, a DPSK modem, a PPM modem, and an optical module controller. Each of the two optical communications terminals will be able to transmit and receive optical signals.

Each optical module, shown in Figure 1, is a 4-inch reflective telescope that produces a $\sim 15$ microradian downlink beam. It also houses a spatial acquisition detector which is a simple quadrant detector, with a field of view of approximately 2 milliradians. It is used both for detection of a scanned uplink signal, and as a tracking sensor for initial pull-in of the signal. The telescope is mounted to a two-axis gimbal stabilized via a magnetohydrodynamic inertial reference unit (MIRU). Angle-rate sensors in the MIRU detect angular disturbances which are then rejected using voice-coil actuators for inertial stabilization of the telescope. Optical fibers couple the optical module to the modems where transmitted optical waveforms are processed. Control for each optical module and its corresponding modems are provided by a controller. Each optical module is held and protected during launch with a cover and one-time launch latch. 


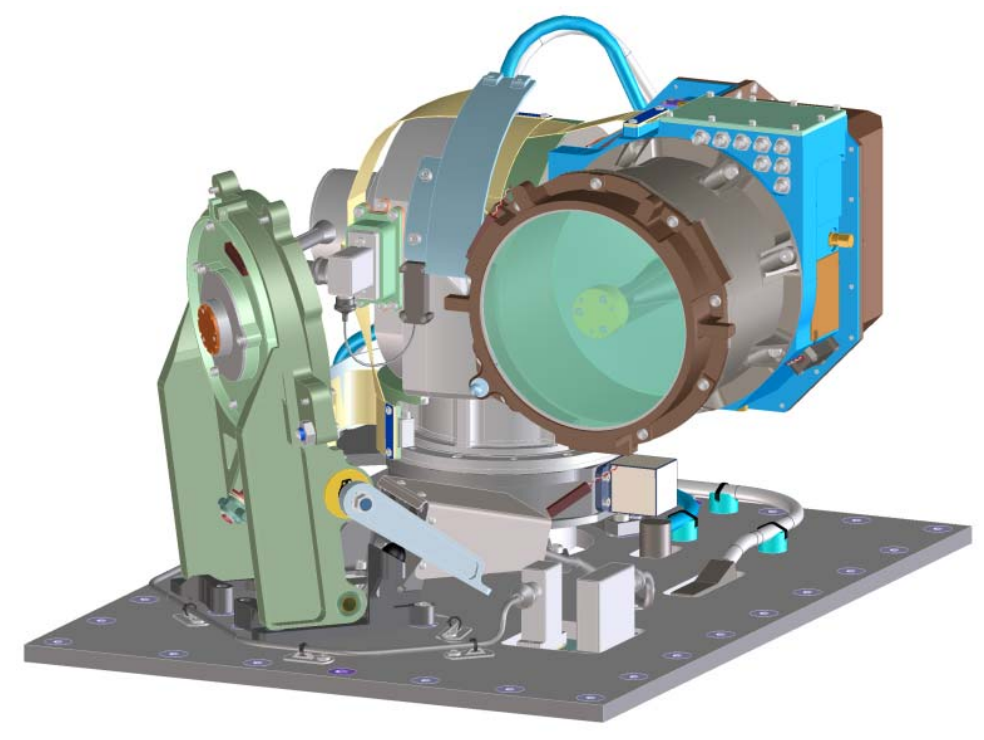

Figure 1- Inertially Stablized Optical Module

Each optical communications terminal supports Differential Phase Shift Keying (DPSK) which has superior noise tolerance, can be used at extremely high data rates, and supports communications when the Sun is in the field of view. LCRD leverages a MIT/LL previously designed DPSK modem [3] as a cost effective approach to providing a DPSK signal. It can both transmit and receive data at an (uncoded) rate from 72 Mbps to 2.88 Gbps. In future relay scenarios, it could be replaced by a higher rate DPSK modem that would support data rates beyond 10 Gbps. Each modem is also capable of supporting Pulse Position Modulation (PPM) at 311 Mbps. PPM can be extremely useful in supporting small disadvantage users, such as small scientific spacecraft. While coding and interleaving will be applied in the ground terminal to mitigate noise and atmospheric fading, the flight receiver does not decode nor de-interleave. The modems instead support a relay architecture where up- and down-link errors are corrected together in a decoder located at the destination ground station [4].

The last critical component of the flight payload is the Space Switching Unit. The Space Switching Unit connects the two optical terminals to allow relay operations. The unit also provides the interface to the host spacecraft.

\section{The Optical Ground Stations}

Each Optical Ground Station (OGS) must provide three functions when communicating with one of the two optical communications terminals on the GEO spacecraft: (1) transmit an uplink beacon beam to support the GEO space terminal in pointing to the correct location on the Earth, (2) transmit a signal beam to the GEO space terminal, and (3) receive the communications signal from the GEO space terminal.

The receiver on Earth must provide a collector large enough to capture adequate power to support the data rate; couple this light onto low noise, efficient detectors while trying to minimize the coupled background light; and perform synchronization, demodulation, and decoding of the received waveform.

The uplink beacon, transmitted from each Earth ground station, must provide a pointing reference to establish the GEO space terminal beam pointing direction. Turbulence effects dominate the laser power required for a ground-based beacon. Turbulence spreads the beam, reducing mean irradiance at the terminal in space, and causes fluctuations in the instantaneous received power.

JPL will enhance its Optical Communications Telescope Laboratory (OCTL) so that it can be used as Ground Station 1 of the demonstration. OCTL is located in the San Gabriel mountains of southern California and houses a 1-m F/76 coudé focus telescope. [5] The large aperture readily supports the high data rate DPSK and PPM downlinks from the LCRD space terminal with adequate link margin. The coude configuration allows for LCRD 
operations to continue during the setup, integration, test and operations of other concurrent experiments. The fullyenclosed design of ground station 1 systems protects users in the coude laboratory, as well as protects the optical systems from accidental jostling and atmospheric disturbances. A fail-safe Laser Safety System at the OCTL (LASSO) will ensure safe laser beam transmission through navigable air and near-Earth space. [6]

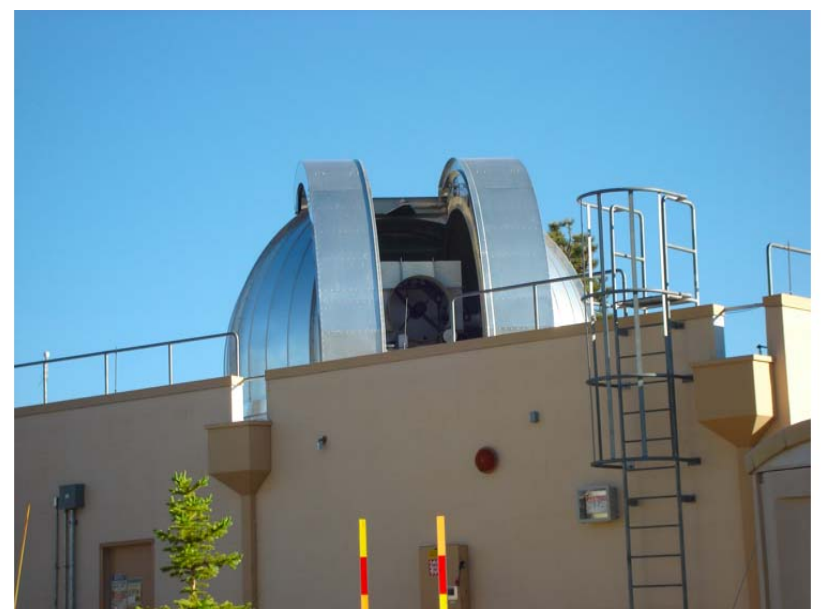

Figure 2 - OCTL telescope will be modified with an optical flat to support links in the presence of more windy conditions.

A state-of-the-art adaptive optics system is being implemented to facilitate coupling the downlinked signal into a single-mode fiber, which conducts it to a high rate detector. The AO system is capable of coupling more than half the received signal into the single mode fiber even under poor seeing conditions and at low elevation angles. A comprehensive monitor and control system will coordinate the receipt of schedules, and direct subsystems to set up and operate according to those schedules. Ground Station 1 also supports extensive simultaneous multi-user, multichannel services such as bitstream, symbolstream, Internet Protocol and AOS. Resident simulators (User MOC Simulators and User Platform Simulators) have been implemented to exercise the system to its full capacity, allowing users to fully characterize the system capability under a wide variety of nominal configurations. An Atmospheric Channel Monitoring system will measure and record real-time data on weather conditions, photometry conditions, and seeing conditions for real-time and subsequent correlation with link performance.

MIT Lincoln Laboratory is developing Ground Station 2 to be deployed somewhere in Hawaii. Ground Station 2 will have a $60 \mathrm{~cm}$ receive aperture, a $15 \mathrm{~cm}$ transmit aperture, and be located within an approximately 5.5 meter diameter dome on one of the islands in Hawaii; ideally it will be located at a summit of one of the volcanoes there to get above the clouds and have excellent atmospheric seeing conditions. NASA has been specifically studying Haleakala, a dormant volcano on the island of Maui, Mauna Kea and Mauna Loa, on the Big Island.
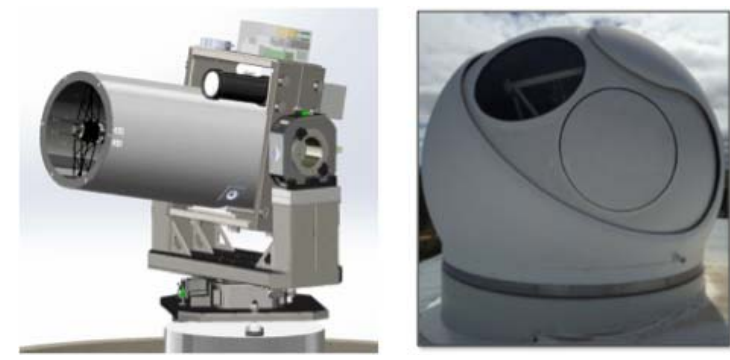

Figure 3 - Ground Station 2 Site Telescope and Dome

Ground Station 2's laser subsystem consists of a custom photonics assembly that produces a low power $(<10 \mathrm{~mW})$ fiber-coupled optical signal, followed by a commercially obtained high-power optical amplifier. The fiber amplifier can produce up to $10 \mathrm{~W}$ of optical power but will be limited by software to a maximum power of $7.3 \mathrm{~W}$ during operation. After accounting for transmission loss of the transmit telescope and the window in the dome, the maximum power emitted into free space outside the dome is $5.4 \mathrm{~W}$. 


\section{Overall LCRD Operations}

LCRD systems are distributed across several facilities to support experiments and demonstrations. LCRD consists of the LCRD Mission Operations Center at WSC, two OGSs located in California and Hawaii, a partial Mission Operations Center (to support experimenters and the engineering team) located at GSFC, and the flight payload in geostationary orbit. The facilities and their locations are shown in Figure 4.

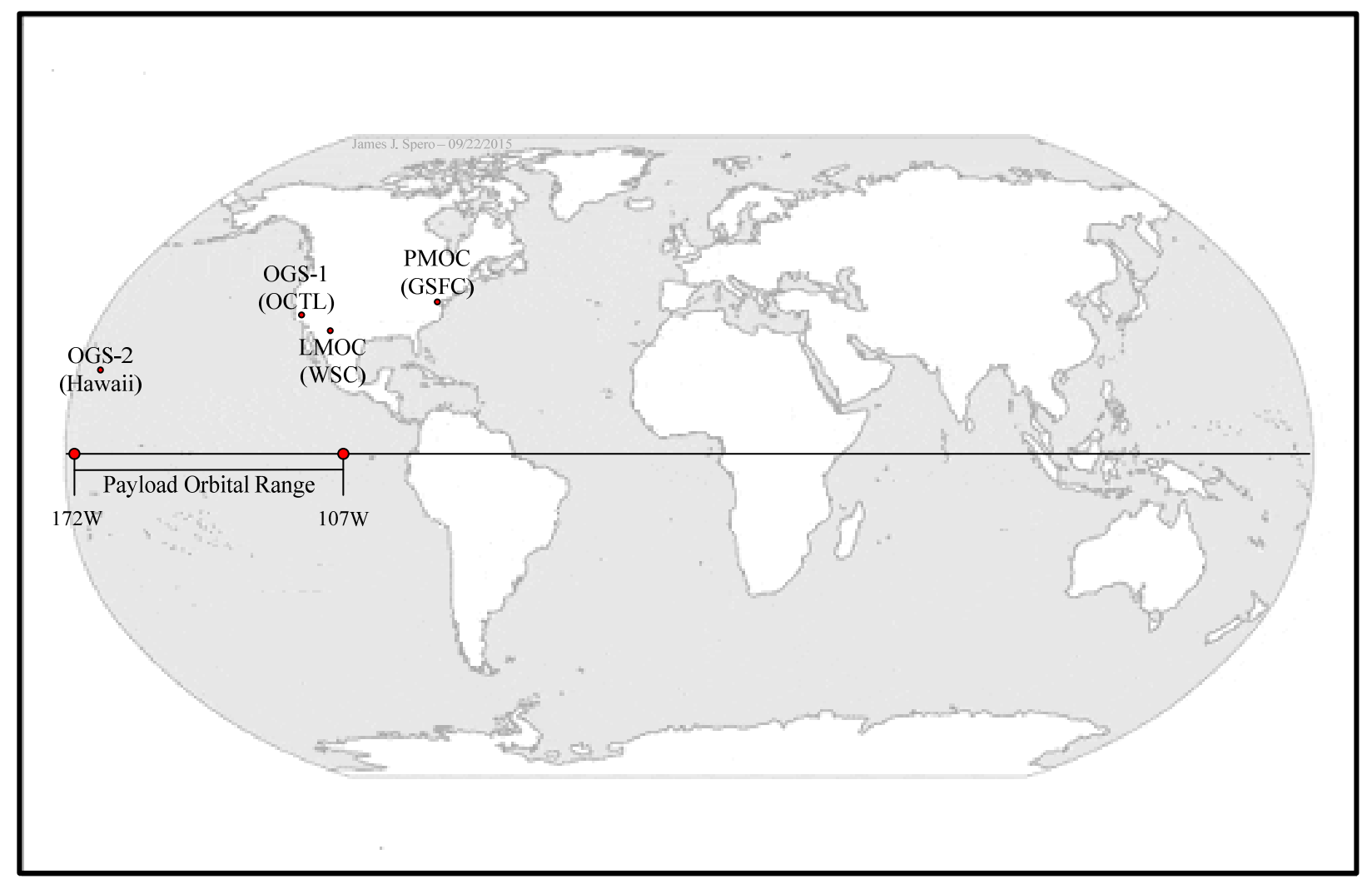

Figure 4 - LCRD Operational Asset Map

Control of all activities during LCRD will take place from the LCRD Mission Operations Center (LMOC). The LMOC is connected with all other segments, and communicates with the two ground stations using high capacity connections. Connection to the space segment will be provided either through one of the ground stations, or through a lower capacity connection to the host spacecraft's Mission Operations Center (HMOC) and then to the LCRD flight payload by RF link.

The LMOC will provide services such as:

- Planning and scheduling

- Control

- Status Monitoring

- Reporting and Accountability

The mission operations for the spacecraft and the optical communications demonstration are intimately intertwined. The unique nature of the demo is that there is a path to and from the spacecraft that is outside the usual RF connection. Commands for the payload can be sent via either the optical uplink or via the Host Spacecraft RF uplink. There are two paths for getting engineering data (health and status), again via optical or RF. The LMOC coordinates all optical communications activities and provides an interface to the spacecraft operations. 
Due to the vagaries of weather and atmospheric conditions, operations strategies for mitigation of these effects will be explored. One possibility would be to have multiple terminals within the same beam simultaneously receive the same data to guarantee getting through to at least one terminal at a reasonably high percentage of the time. On the other hand, buffering and retransmission strategies can be used to downlink the data to single geographically (and meteorologically) diverse stations in a form of temporal diversity.

The ground stations will have the capability to simulate both user spacecraft and user MOC data systems. This will allow the demonstration of high data rate scenarios without the requirement for high data rate connections external to the ground stations. The simulators will also allow multiple user and user-type scenarios. The LCRD payload itself will also include the ability to simulate user spacecraft data and multiple relay user spacecraft data systems.

The system will be continuously operating, as much as possible, over the two year mission. The system will either be configured to be demonstrating or testing a specific Direct-to-Earth (DTE) scenario, relay scenario, or be continually characterizing the optical channel and hardware. The DTE and relay scenarios will emulate different user and relay locations, orbits, and/or trajectories.

\section{Flight Payload Operations}

The LCRD flight payload has the following operating modes as defined in Figure 5:

1. Off/Survival Mode: In this mode the LCRD Payload power is removed and only the survival heaters are powered from the Host Spacecraft. The Payload will launch in this mode and remain there until the first poweron event.

2. Payload System Initialization Mode: In this mode, the Payload is powered on, but not yet ready to support normal operations. The Payload will enter this mode in one of two cases: Payload initial power up after launch or Payload return to power after Off/Survival mode.

3. Payload Self-Test/Calibration Mode: In this mode, the Payload will have at least the SSU and one OST powered on. The second OST may be either powered off or powered on. This mode is used to execute predefined self-test functions within the Payload.

4. Payload Operational Mode: In this mode, the Payload has been powered up and initial configuration established such that the Payload can communicate with the LMOC via the Host interface, can attempt to acquire an optical communication links with OGSs and/or User platforms, and once a link is established provide forward and return service relays and Direct Uplinks/Downlinks.

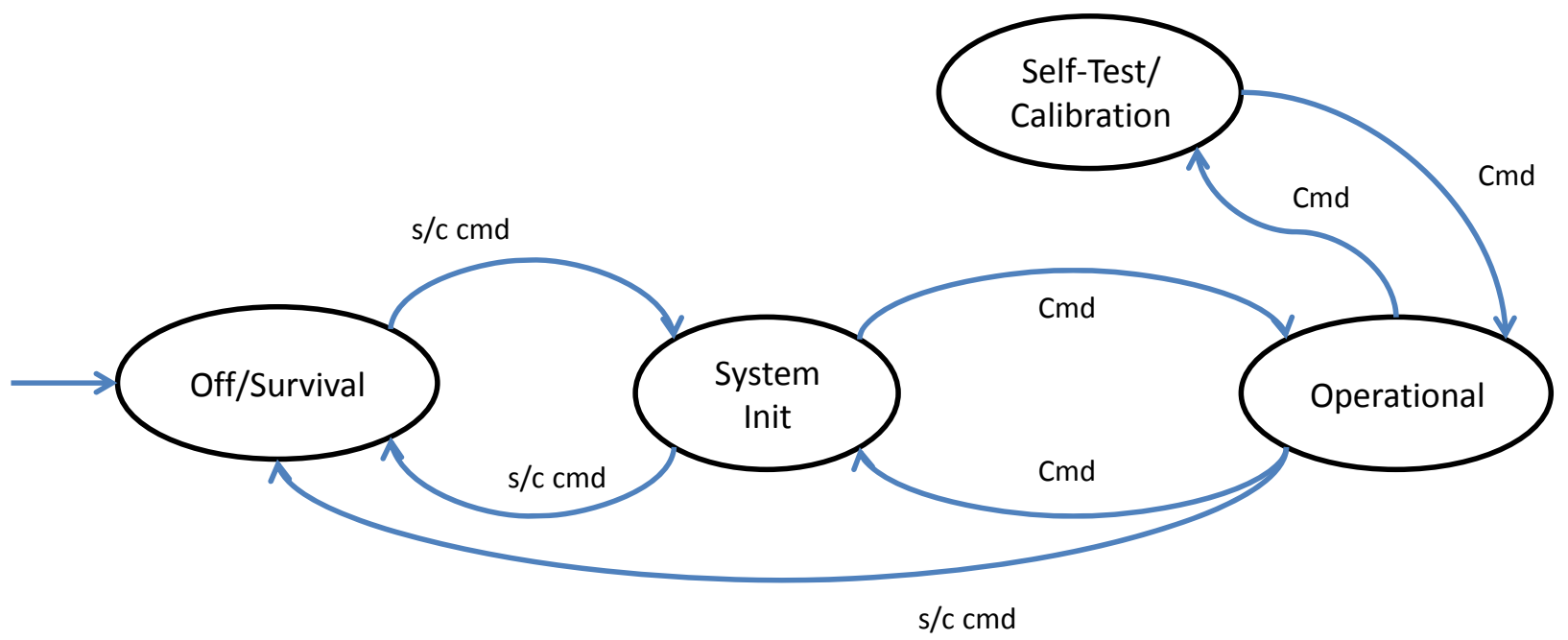

Figure 5 - LCRD Payload Mode Transition Diagram 


\section{Ground Segment Operations}

The ground segment consists primarily of the LMOC and two OGSs. The LMOC coordinates all LCRD operations activities, provides the mission data store, provides an interface for User service planning and scheduling, and provides the experiment operations center. The OGSs provide a User MOC interface, User MOC and user platform simulators, and the optical link from the Earth to the Payload. The LMOC will operate 24 hours per day, seven day per week to receive Payload, OGS, and Host Mission telemetry. The LMOC and OGSs will initially be staffed 8 hours per day, 5 days per week after on-orbit Payload checkout to conduct experiments and support User services. The ground segment will be staffed for certain 24 hour period during the mission to support longer duration experiment operations.

Routine operations will be performed continuously during staffed operations periods. The main operations tasks will be performed from the LMOC at WSC and include:

- Monitor payload and ground system telemetry for proper execution of commands

- Monitor payload and ground system telemetry for limit violations or changes in state that may be a precursor to limit violations

- Monitor payload and ground system telemetry for anomalies that threaten health and safety, and to take appropriate action to ensure health and safety in the event of a system anomaly

- Monitor the atmospheric conditions at each ground station

- Monitor link status for each optical link and the host spacecraft RF link

- Monitor quality of service for each user service

- Respond to user service requests and support requests from the ExpO team and external users

- Perform planned and unplanned ground station handover and ground system reconfiguration operations when required

- Execute performance data monitoring functions and data storage in support of ExpO team analysis

- Implement scheduling change requests from the ExpO team and external users

- Perform routine housekeeping functions including data store back up, periodic payload and ground calibration functions, clock management, data correlation, preventive system maintenance and other planned maintenance and repair activities.

- Contact engineering, ground system, or supervisory personnel in the event of a significant anomaly

Each LCRD OGS will be staffed during the same period as the LMOC to support routine operations which include:

- Provide laser safety functions

- Monitor and control ground station local equipment

- Monitor the atmospheric conditions at the ground station

- Monitor link status for the ground station optical link

- Monitor quality of service for each User service provided at the ground station

- Support diagnostic procedures when degraded performance is detected

- Perform routine housekeeping functions including data store back up, ground station optical calibration functions, clock management, preventive system maintenance and other planned maintenance activities

- Contact engineering, ground system, or supervisory personnel in the event of a significant anomaly

\section{Pointing, Acquisition, and Tracking Concept of Operations}

There are two Pointing Acquisition, and Tracking (PAT) operations cases for LCRD. The first is the establishment of optical links between the payload and an OGS. This is the simplest case given the relative stability of the host spacecraft orbit and the fixed location of the ground stations on the Earth's surface. This case will generally be applied to at least one optical terminal on the payload for normal operations. It may be applied to both optical terminals during demonstration and experiment activities and may include an outside user ground station not under LCRD control. The second case is PAT operations for a moving user platform. This could include an airborne platform or space-borne Low Earth Orbit (LEO) platform (up to $1000 \mathrm{~km}$ altitude). The most complex case for LCRD will be PAT associated with a LEO platform due to relative movement of the LEO platform with regard to the payload. This case may be applied to at least one optical terminal on the payload to support a user forward 
and/or return relay service. It may be applied to both optical terminals during demonstration and experiment activities to support user-to-user relays.

The LCRD payload, each LCRD OGS, and user platforms will follow a predefined acquisition sequence. The LMOC will coordinate the initial acquisition sequence between the payload and a ground station element and/or the payload and a user platform. A series of states are passed through during the acquisition process. Each state represents an intermediate step that is the result of a prior action. The diagram illustrating nominal acquisition sequence flow from state to state is shown in Figure 6. In this diagram states evolve from left to right during the course of the acquisition.

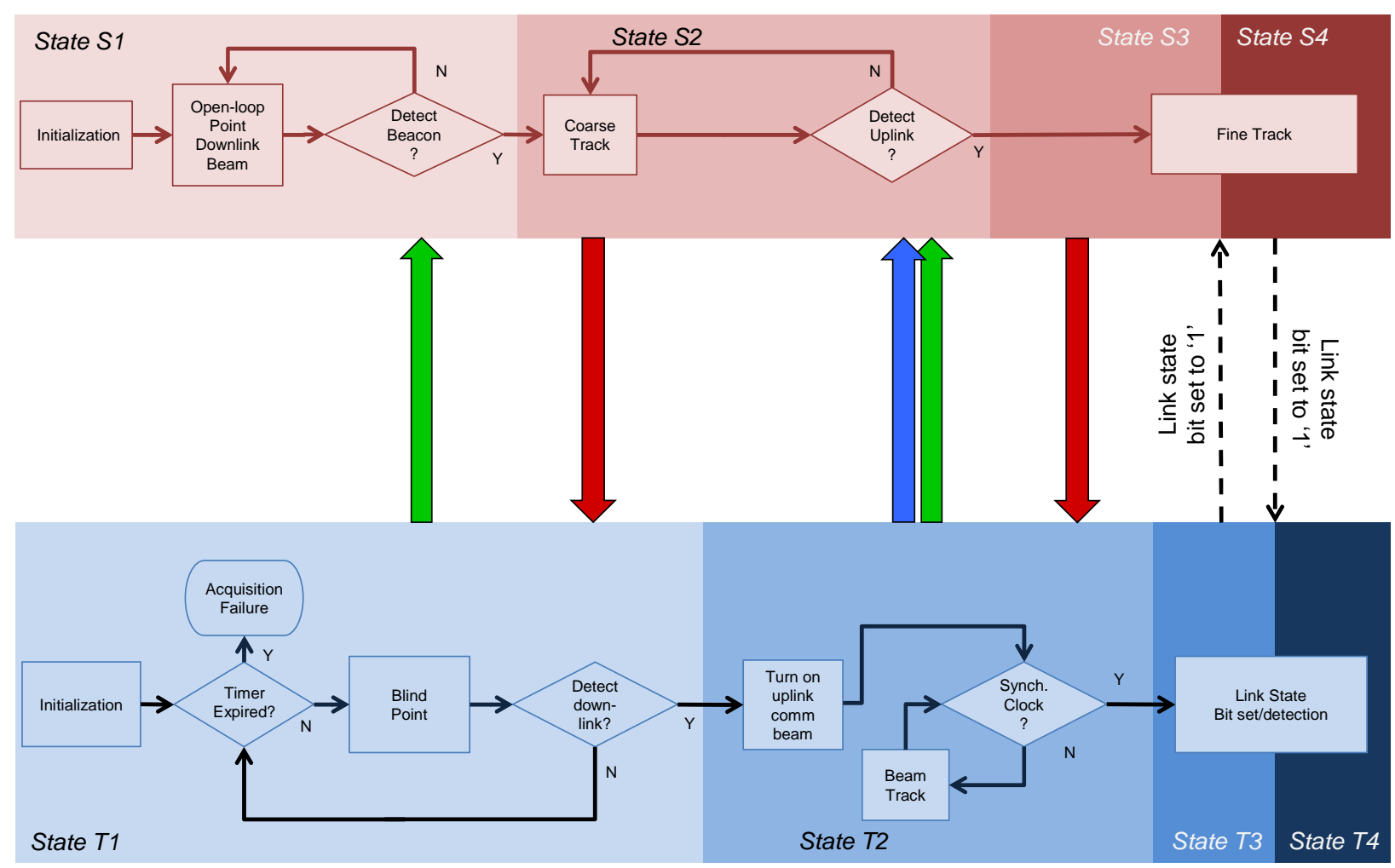

Figure 6 - Nominal LCRD Acquisition Flow

The LCRD Payload Optical Space Terminal states are:

- S1 - LCRD Optical Space Terminal initialized but not illuminated by the beacon or uplink beam

- S2 - LCRD Optical Space Terminal illuminated by the beacon

- S3 - LCRD Optical Space Terminal illuminated by the uplink beam

- S4 - LCRD Optical Space Terminal receiving data frames

The Ground Stations or User Platform states are:

- T1 - Ground Station or User Platform initialized but not illuminated by the downlink

- T2 - Ground Station or User Platform illuminated by the (optionally dithered) downlink beam

- T3 - Ground Station or User Platform synchronized clock to downlink beam stream

- T4 - Ground Station or User Platform receiving data frames

Once initial acquisition has been established between an OGS and the payload or the payload and a user platform, maintaining the communications link is handled by the payload and the OGS or the payload and the user without interaction from the LMOC. The payload and OGSs may use a Closed Loop Point Ahead (CLPA) algorithm built into the optical link to maintain accurate pointing. The link between the payload and a user platform is required to use CLPA. The User platform is required to apply Doppler compensation to maintain the link between the Payload and the User platform. 


\section{Optical Trunk Line Concept of Operations}

LCRD provides the capability to multiplex user service data and payload command and telemetry data using the same optical link. This is referred to as an "optical trunk line" and is illustrated in Figure 7. The trunk line concept allows multiple users, types of data, and data destinations to be multiplexed together on the optical link at the optical frame level. The forward data trunk line is demultiplexed at the LCRD payload, and user data or commands routed to the correct user platform (or executed by the payload in the case of command). The return trunk line data frames get demultiplexed on the ground; User data sent to the appropriate user MOC, and telemetry is sent to the LCRD MOC.

The optical trunk line can support a slot rate of $2.88 \mathrm{Gbps}$ in each direction for any modulation or data rate. The optical frame rate depends upon the modulation (DPSK or PPM), configured link dead time, and number of Qrepeats. The transmit side modem sends a continuous stream of frames. LCRD supports multiplexing of multiple data streams over a single optical link in both directions: user service data frames, payload command and telemetry frames, and idle fill frames (sent whenever there is no user data or payload commands or telemetry). This is achieved by the use of dynamic Time Division Multiple Access (TDMA), with the frames sent "on demand", in the order that they are received by the modem.

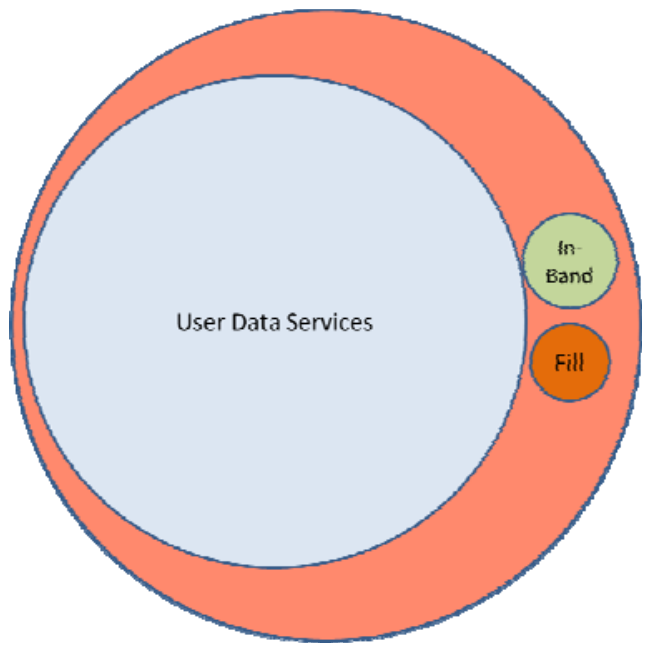

Figure 7 - Optical Trunk Line

\section{Ground Station Handover Concept of Operations}

Under certain conditions, the optical link between the payload and an OGS may be handed over to another OGS. A handover between ground stations may only occur if there is an available ground station to receive the handed over optical link. Some conditions that may drive this scenario are weather, laser safety issues, planned or unplanned maintenance, and laser operation blackout periods. Handovers are a highly manual procedure with no autonomy built into the payload. All handover operations must be coordinated through the LMOC, whether planned or unplanned. The general sequence of events is the same for both planned and unplanned handover scenarios. In this context, "OGS-A" is the station handing over, "OGS-B" is the receiving ground station. Assume OGS-B is fully functional with locally nominal atmospheric conditions.

1. User(s) and LCRD assets notified of impending ground station handover if possible.

2. OGS-B brought to operational/hot stand by state. This state means being configured to begin the acquisition sequence with the Payload and in the same optical link configuration (data rate an modulation) and ground system configuration as OGS-A for user services and in-band command/telemetry.

3. User services and/or in-band command/telemetry over the optical link terminated at OGS-A and the Payload.

4. Users establish forward/return data link with OGS-B and terminate with OGS-A.

5. Payload is configured via the host spacecraft RF link to point an OST at OGS-B and is ready to begin acquisition.

6. OGS-B and the payload complete acquisition sequence and establish optical communications link. 
7. User services and/or in-band command/telemetry resume through OGS-B.

Figure 8 illustrates the pre-handover configuration and the post-handover configuration. During this scenario, the optical link with the user platform does not have to be terminated and should remain established through the second OST if possible to expedite resumption of service. The user will lose any data transmitted during the ground station handover. The user will be notified ahead of time if possible to mitigate data loss if the user mission desires.

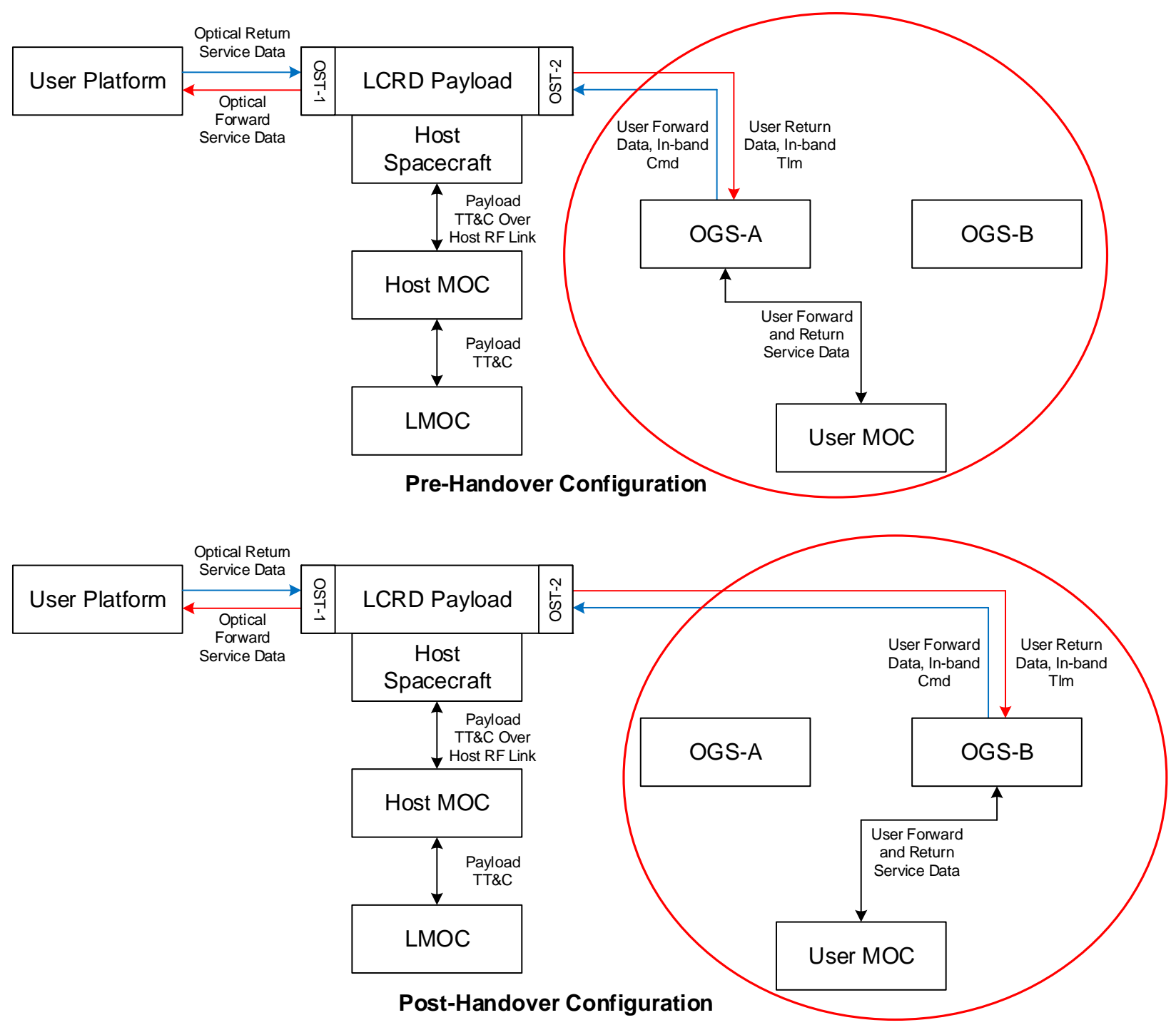

Figure 8 - Optical Ground Station Handover

\section{Conducting Experiments}

LCRD is designed to support demonstrations and experiments in optical communications and optical communication networking. The LCRD Principle Investigator will lead a team to define demonstrations and experiments to be executed using LCRD mission assets. As the mission progresses, new demonstrations and experiments will be defined and executed using the base system functions or system enhancements.

LCRD will support simulation of:

1. User-GEO-Ground relay

2. User-to-User Relay 
3. Near Earth and Deep Space Relay

4. Direct Uplink/Downlink

5. Optical trunk line Handovers

6. Forward/return link handovers

LCRD will enable the following capabilities required to support experiments and demonstrations:

1. User MOC Simulators (UMSs) and User Platform Simulators (UPSs)

2. Data collection and tools for analysis of data and link characteristics

3. Simulation of various latencies in data transmission and receipt

4. Variations in scheduled service data rates and combinations of service data rates

5. Data rate variations in response to deteriorating link conditions

6. Variations in user traffic loading relative to allocated service data rates

7. Simulation of weather and atmospheric effects

8. Simulation of different line-of-site distances

9. Variations in relay asset availability

Laser transmitter power reduction from Ground Stations, off-pointing the payload optical space terminal or attenuating the signal at the Ground Station may be used to simulate the effects of the channel on the weak signals returned from deep space distances. The relay may also use different modulation or data rates on each leg to simulate collection of data from multiple sources at lower data rates, which is then combined and relayed to a Ground Station.

Relay tests in each Near-Earth or deep space scenario will attempt to simulate differences in latency, maximum data rates, line-of-site and scheduled link availability, and other required tuning to accurately reflect different scenarios. LCRD will enable the implementation of tools required to support these scenarios over the course of the mission. The LCRD system will enable evaluation of protocols such as Disruption Tolerant Networking (DTN), which are meant to alleviate the related communications issues.

\section{Scheduling Service}

The LMOC is responsible for coordination of LCRD network activities. The LMOC is the focal point for planning the use of LCRD resources for functions including experiment operations, user service support, payload operations, sustaining engineering activities, and maintenance activities. The LMOC will be responsible for coordinating the configuration of LMOC subsystems, the LCRD payload, and OGS support.

The LMOC will produce three schedule types covering different timescales: the Mission Timeline, the Active Schedule, and the Operational Schedule. The Mission Timeline will be a human-readable, high-level, long-term operations schedule incorporating long-range planning inputs. Its purpose will be to allow each ground element to plan their operator staffing schedules, to arrange periods of extended operations (outside of typical operating times), and to identify potential scheduling conflicts well in advance to facilitate negotiating solutions. Inputs will include scheduled LMOC downtimes, availability schedules for each OGS, availability of external interface systems, highlevel experiment plans, and LCRD User Service Level Agreements.

The Active Schedule will be the medium-term planning product consisting of the conflict-free, constraint checked, and optimized schedule of committed operational services and all LCRD activities necessary to support those services. Its purpose will be to represent the configuration of all LCRD assets and support schedules or constraints from external entities to ensure a consistent configuration that will enable execution of planned user services. Inputs will include the current Mission Timeline, updates to scheduled LMOC downtimes, updates to availability schedules for each OGS, availability of external interfaces, predictive avoidance constraints from the Laser Clearing House, detailed experiment plans, and user service requests. Mission Planning and Scheduling will generate an updated Active Schedule a minimum of once per week for LCRD operations covering the next fourteen 24-hour operational days.

Mission Planning and Scheduling will generate two additional types of products for external systems based on the Active Schedule. The first will be a predictive avoidance request message covering the next few days of laser 
activity by the LCRD payload optical terminal that will be sent to the Laser Clearing House. This will be used by Laser Clearing House to generate a Predictive Avoidance file for LCRD. The second product for an external system will be a high-level activity schedule containing times the LCRD payload will operate and any operational events that may impact host spacecraft flight or ground operations. The LMOC will send this schedule to the Host Mission Operations Center as a planning aid for the host spaceccraft mission.

The Operational Schedule will be the short-term schedule provided to the LMOC subsystems to be executed for asset configuration and control. This schedule is generated once before every "operational day", which is defined to be the current 24-hour period beginning at 00:00:00 UTC. It will facilitate timing and coordinating the configuration of all assets to support user services and experiments. This schedule may be regenerated at any time during an operational day for unplanned OGS handovers, unplanned maintenance or downtime events for the LMOC, LCRD payload, each OGS, or external support assets, changes to experiment or demonstration support plans, change requests from users, or any other reason.

An Operational Schedule Excerpt (OSE) will be generated from the full Operational Schedule and distributed to each asset required to support the schedule for the current operational day. The OSE consists of the subset of the full Operational Schedule that pertains specifically to that asset, will be executable by that asset's local control system, and will contain any information required to execute the schedule. The appropriate versions will be sent to the LMOC Service Management subsystem, Payload T\&C System, and each OGS. An Operational Schedule for each actual or simulated user will also be generated representing the timing and duration of the user platform optical link and User Mission Operations Center ground connection and the traffic profiles associated with each scheduled data service for the latter. If the Operational Schedule is regenerated for a given operational day, a new set of OSEs will be generated and distributed.

LCRD will ingest planning aids and information from external sources for use in conflict resolution and optimization of the Active Schedule and Operational Schedule. These products include the Predictive Avoidance file from the Laser Clearing House, planning aids from the host spacecraft operator, and flight dynamic information from LCRD users.

Planning for a given time period will begin with the process of adding that period to the Mission Timeline. This will facilitate long-term planning, principally resolving issues of staffing and asset downtime, and will be largely a manual process carried out by the mission planner. In the medium term the mission planner will add activities for the period to the active schedule. This will allow a more detailed representation of planned activities in software and conflict resolution and optimization of the schedule. Near the start of a given operational day, the Mission Planning and Scheduling function will output the Operational Schedule covering that operational day based on the current Active Schedule.

\section{Conclusion}

NASA science and human exploration missions continue to seek ever higher instantaneous data rates and/or data volume delivery. While, the capacity of current and near-term RF communications technology is still increasing, it is becoming significantly harder and more expensive to increase performance. RF communications is limited by bandwidth allocation restrictions, power requirements, flight terminal antenna size, and weight limitations. The cost and complexity of expanding the existing Space Communications Networks to enable these higher data rates using RF solutions with large aperture antennas is a significant undertaking. Optical communications holds the potential for high data rates with smaller systems on user spacecraft and on the ground.

NASA's Laser Communications Relay Demonstration will demonstrate continuous high data rate optical communications in an operational environment, demonstrating how optical communications can meet NASA's growing need for higher data rates. LCRD will serve as a developmental testbed in space and for gaining confidence in this new technology. The project is a critical stepping stone towards the Next Generation Tracking and Data Relay Satellite, as we believe strongly that the next generation satellite will supply both RF and optical services. Doing this demonstration will allow initial operational capability (IOC) of an optical service on the first next generation satellite. 


\section{Acknowledgments}

The work described in this paper was carried out at NASA's Goddard Space Flight Center; at Lincoln Laboratory*, Massachusetts Institute of Technology; and at the Jet Propulsion Laboratory, California Institute of Technology, under contract with NASA. It is funded by NASA's Space Technology Mission Directorate and NASA's Space Communications and Navigation Program Office.

\section{References}

1. B. L. Edwards, D. J. Israel, K. E. Wilson, J. Moores, A. Fletcher, "Overview of the Laser Communications Relay Demonstration Project”, Space Operations Conference, June 2012

2. B. L. Edwards, D. J. Israel, “A Geosynchronous Orbit Optical Communications Relay Architecture”, IEEE Aerospace Conference, March 2014

3. Caplan, D.O.; Rao, H.; Wang, J.P.; Boroson, D.M.; Carney, J. J.; Fletcher, A.S.; Hamilton, S.A.; Kochhar, R.; Magliocco, R.J.; Murphy, R.; Norvig, M.; Robinson, B.S.; Schulein, R.T.; Spellmeyer, N.W.; , "Ultra-wide-range multi-rate DPSK laser communications," Lasers and Electro-Optics (CLEO) and Quantum Electronics and Laser Science Conference (QELS), 2010 Conference on , vol., no., pp.1-2, 16-21 May 2010

4. Shoup, R.; List, N.; Fletcher, A.; Royster, T.; , "Using DVB-S2 over asymmetric heterogeneous optical to radio frequency satellite links," MILITARY COMMUNICATIONS CONFERENCE, 2010 MILCOM 2010 , vol., no., pp.785-790, Oct. 31 2010-Nov. 32010

5. K. E. Wilson, J. Wu, N. Page, M. Srinivasan, "The JPL Optical Communications Telescope Laboratory (OCTL), Test Bed For The Future Optical Deep Space Network” JPL, Telecommunications and Data Acquisition Progress Report 142 -153 February 2003.

6. K. Wilson, W.T. Roberts, V. Garkanian, F. Battle, R. Leblanc, H. Hemmati, and P. Robles "Plan For Safe Laser Beam Propagation From The Optical Communications Telescope Laboratory” JPL, Telecommunications and Data Acquisition Progress Report 142 -152, February 2003.

7. Grein, M.E.; Kerman, A.J.; Dauler, E.A.; Shatrovoy, O.; Molnar, R.J.; Rosenberg, D.; Yoon, J.; DeVoe, C.E.; Murphy, D.V.; Robinson, B.S.; Boroson, D.M.; , "Design of a ground-based optical receiver for the lunar laser communications demonstration," Space Optical Systems and Applications (ICSOS), 2011 International Conference on , vol., no., pp.78-82, 11-13 May 2011

\footnotetext{
* This work was sponsored by NASA Goddard Space Flight Center under Air Force Contract FA8721-05-C-0002. The opinions, interpretations, conclusions, and recommendations are those of the author and not necessarily endorsed by the United States Government.
} 